\title{
Optical trapping and integration of semiconductor nanowire assemblies in water
}

\author{
PETER J. PAUZAUSKIE ${ }^{1}$, ALEKSANDRA RADENOVIC ${ }^{2,3}$, ELIANE TREPAGNIER ${ }^{2,3,4}$, HARI SHROFF',3,4, \\ PEIDONG YANG ${ }^{1,5 *}$ AND JAN LIPHARDT2,3,4*
}

\author{
${ }^{1}$ Department of Chemistry, University of California, Berkeley, California 94720, USA \\ ${ }^{2}$ Department of Physics, University of California, Berkeley, California 94720, USA \\ ${ }^{3}$ Physical Biosciences Division, Lawrence Berkeley National Laboratory, Berkeley, California 94720, USA \\ ${ }^{4}$ Biophysics Graduate Group, University of California, Berkeley, California 94720, USA \\ ${ }^{5}$ Materials Science Division, Lawrence Berkeley National Laboratory, Berkeley, California 94720, USA \\ *e-mail: p_yang@berkeley.edu; liphardt@physics.berkeley.edu
}

$(0)$ emiconductor nanowires have received much attention owing to their potential use as building blocks of miniaturized electrical ${ }^{1}$, nanofluidic ${ }^{2}$ and optical devices ${ }^{3}$. Although chemical nanowire synthesis procedures have matured and now yield nanowires with specific composition ${ }^{4}$ and growth directions $s^{5}$, the use of these materials in scientific, biomedical and microelectronic applications is greatly restricted owing to a lack of methods to assemble nanowires into complex heterostructures with high spatial and angular precision. Here we show that an infrared single-beam optical trap can be used to individually trap, transfer and assemble high-aspect-ratio semiconductor nanowires into arbitrary structures in a fluid environment. Nanowires with diameters as small as $20 \mathrm{~nm}$ and aspect ratios of more than 100 can be trapped and transported in three dimensions, enabling the construction of nanowire architectures that may function as active photonic devices. Moreover, nanowire structures can now be assembled in physiological environments, offering new forms of chemical, mechanical and optical stimulation of living cells.

At present, several nanowire assembly techniques are being investigated, including electric ${ }^{6}$ and magnetic ${ }^{7}$ fields, laminar flow in microfluidic channels ${ }^{8}$, and Langmuir-Blodgett compression ${ }^{9}$. Although these techniques can align groups of nanowires, they lack the ability to control and assemble individual wires into two- or three-dimensional heterostructures ${ }^{10}$. Optical traps are an appealing tool for semiconductor nanowire integration owing to their ability to act in situ in closed aqueous chambers, their potential applicability to a broad range of dielectric materials, their spatial positioning accuracy ${ }^{11}(<1 \mathrm{~nm})$, and the degree to which their intensity, wavelength and polarization can be controlled using tuneable lasers, acousto-optic modulators and holographic optical elements ${ }^{12}$. Single-beam optical traps have been used for almost two decades $^{13}$ to manipulate and interrogate objects of micrometre and nanometre size ${ }^{14}$. In 1994, optical confinement of metal particles in two dimensions was achieved ${ }^{15}$ and it was shown ${ }^{16}$ that a 36-nm-diameter gold particle could be optically trapped in three dimensions. More recently, birefringent crystals were rotated in an optical trap by angular momentum transfer ${ }^{17}$, and $\mathrm{CuO}$ nanorods were manipulated in two dimensions with a line optical trap ${ }^{18}$.

In the Mie limit, applicable to objects much larger than the wavelength of light, ray-tracing can be used to calculate the forces exerted on objects by an optical potential ${ }^{19,20}$. Optical trapping of objects much smaller than the wavelength of light, such as certain glass nanorods, is theoretically tractable using the standard framework of the Rayleigh limit ${ }^{21,22}$. However, nanowires targeted for use in integrated optical and electrical circuits usually have aspect ratios larger than 100 and diameters smaller than $80 \mathrm{~nm}$ (two dimensions in the Rayleigh limit, the third in the Mie limit), may have asymmetrical cross-sections, non-zero absorption and typically have poorly characterized polarizabilities. These properties of semiconductor and metallic nanowires make it difficult to model their confinement by an optical potential. Therefore, we first determined whether a range of semiconductor nanowires could be stably trapped with a simple single-beam optical trap.

Trapping experiments were performed in a purpose-built single-beam optical-tweezers system (see Fig. 1a and Supplementary Information, Fig. S1). Nanowires were suspended in water and the suspension was transferred by means of a pipette into a chamber created with $100-\mu \mathrm{m}$-thick double-sided tape between the glass and quartz coverslips (Fig. 1c). Once sealed inside the chamber, the nanowires sank to the bottom. When the laser trap was brought close to $\mathrm{GaN}, \mathrm{SnO}_{2}, \mathrm{ZnO}$ or Si nanowires, they were pulled into the trap. The $\mathrm{ZnO}$, Si and circular cross-section $\mathrm{GaN}$ wires trapped stably, with one end of the wire in the laser focus and the rest of the wire parallel to the optical axis (Fig. 1c). Wires were not 


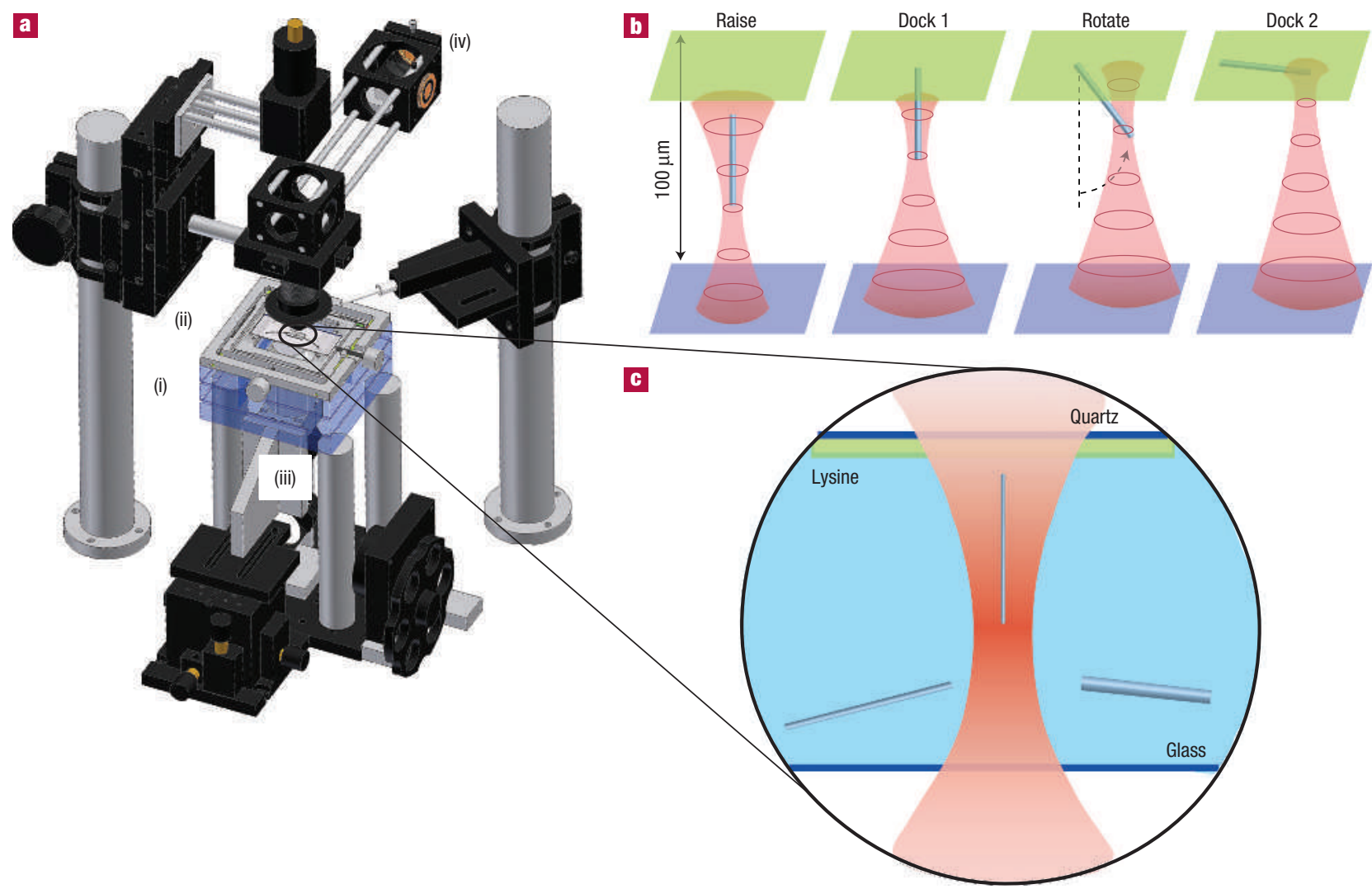

Figure 1 Setup for the trapping experiments and the procedure for nanowire docking at a surface. a, Schematic of optical trapping instrument. (i) Three-axis piezoelectric positioning stage. (ii) Custom-built coarse-movement translation stage. (iii) Objective holder. (iv) PSD. b, Schematic of the four-step nanowire positioning procedure. c, Schematic of the experimental chamber cross-section. The top surface consists of a 170- $\mu \mathrm{m}$-thick synthetic fused silica coverslip (blue) coated with lysine or gold (green). The bottom surface consists of a standard number 1 thickness rectangular glass coverslip. Owing to gravity, free nanowires sink to the bottom surface, where they can be picked up with the optical trap.

only confined in two dimensions, but in all three dimensions, which allowed controlled assembly. As the wires were confined in all three dimensions, it was possible to pick them up from the bottom chamber surface and to bring them to the top chamber surface (at least $100 \mu \mathrm{m}$ away). The free nanowires at the lower surface were thus well-separated from the top surface, where nanowire characterization and assembly took place. We found that the easiest way to pick up nanowires was by aiming the laser trap at one of the nanowire's ends and then translating the trap along the wire, simultaneously raising the trap. This motion of the optical trap with respect to the wire lifted and rotated the wire perpendicular to the surface. Once the nanowire was trapped stably, translation was performed with either a home-built translation stage for long (millimetre) distances or a three-axis piezo-stage for fine (nanometre) control. Velocities of $10 \mu \mathrm{m} \mathrm{s}^{-1}$ were routinely achieved in all three axes, as were millimetre-scale translations. We did not observe aggregation of semiconductor nanowires in our optical trap, presumably because free nanowires quickly sank to the bottom coverslip surface, and because a nanowire must be aligned with the trap's optical axis for stable confinement.

Despite their lack of three mutually orthogonal symmetry planes, GaN nanowires with isosceles cross-sections also trapped stably, in part owing to their subwavelength cross-section (as low as $20 \mathrm{~nm}$ ). However, about $6.5 \%$ of 200 tin oxide ribbons showed unexpected periodic oscillations (see Fig. 2a and Supplementary Information, Fig. S2). About 5\% oscillated between two states (see Supplementary Information, Video S1) and a further 1.5\% visited as many as six different states per cycle (see Fig. 2a and Supplementary Information, Video S2). The oscillation period was a linear function of the laser power (see Supplementary Information, Fig. S2a). Further analysis revealed that the most complex oscillations are related to the asymmetric cross-section of coupled $\mathrm{SnO}_{2}$ ribbons (see Supplementary Information). In contrast to the semiconducting wires, silver nanowires were not stably confined and also showed significant thermal instability (see Supplementary Information, Fig. S3). The observed behaviours of the nanowires in contact with the laser trap thus ranged from no trapping (silver), stably trapped but oscillating (some $\mathrm{SnO}_{2}$ ) and stably trapped with no oscillations $\left(\mathrm{GaN}, \mathrm{ZnO}\right.$, Si and most $\left.\mathrm{SnO}_{2}\right)$; see Table 1 .

Judging from the ease of wire pick-up and the maximum wire translation velocity $\left(\sim 10 \mu \mathrm{m} \mathrm{s}^{-1}\right), \mathrm{GaN}, \mathrm{Si}$ and $\mathrm{SnO}_{2}$ have similar trapping properties. $\mathrm{ZnO}$ wires, on the other hand, are easier to pick up and can be translated at velocities greater than $20 \mu \mathrm{m} \mathrm{s}^{-1}$ without escaping from the trap. We further characterized the $\mathrm{GaN}$ wires as their trapping properties are similar to $\mathrm{Si}$ and $\mathrm{SnO}_{2}$, they possess a particularly large aspect ratio and two cross-sections are available. The power spectrum of trapping light collected on a position-sensitive detector (PSD) was well-fit by a lorentzian (Fig. 2b, inset) whose corner frequency depended on the laser power, and the GaN wire's cross-section and aspect ratio (Fig. 2b). At a laser power of $60 \mathrm{~mW}$, cylindrical GaN nanowires with a 
Table 1 The geometrical characteristics, physical properties and trapping behaviour of nanowires.

\begin{tabular}{llllll}
\hline Material & Cross-section & Length $(\mu \mathrm{m})$ & Diameter $(\mathrm{nm})$ & Trappable? & Refractive index at 1,064 $\mathrm{nm}$ \\
\hline $\mathrm{GaN}$ & $\circ$ & $1-100$ & $20-200$ & Yes & 2.26 \\
$\mathrm{GaN}$ & $\triangle$ & $1-100$ & $20-200$ & Yes & 1.94 \\
$\mathrm{SnO}{ }_{2}$ & $\square$ & $1-100$ & $200-600$ & Yes* & 1.96 \\
$\mathrm{ZnO}$ & $\square$ & $1-50$ & $30-60$ & Yes & 3.6 \\
$\mathrm{Si}$ & $\square$ & $1-15$ & $10-20$ & Yes & 6.1 \\
$\mathrm{Ag}$ & $\square$ & $1-15$ & 50 & No & \\
\hline
\end{tabular}

${ }^{\text {* } \mathrm{About}} 7 \%$ of trapped $\mathrm{SnO}_{2}$ wires oscillated in the trap.

$50 \mathrm{~nm}$ radius and about $20 \mu \mathrm{m}$ length were confined by an optical potential with a spring constant of around $10^{-3} \mathrm{pN} \mathrm{nm}^{-1}$, about 10-100-fold less than a micrometre-sized polystyrene bead.

Assembly of complex nanowire structures requires not only manipulation of individual wires, but also the controlled connection of one wire to another. We observed that it is possible to locally fuse two wires by way of a focused infrared beam. On laser irradiation of the crossing point of two wires for $5 \mathrm{~s}$ using higher power $(\sim 1 \mathrm{~W})$ than for trapping, the two wires stopped moving with respect to one another and could not be pulled apart, presumably because they had been fused at the crossing point. On the basis of a simple order-of-magnitude calculation $^{23}$, it is possible that local temperatures at the junction can approach the melting point of $\mathrm{GaN}$ and $\mathrm{SnO}_{2}$. Thermal fusing is consistent with our electron-microscopy investigations, which demonstrated that nanowires can be melted and welded at temperatures lower than required for bulk materials ${ }^{24}$. In addition, at the highest powers, water vapourized into small bubbles, limiting the maximum intensity used in forming the connection because of perturbations from the bubble. Scanning electron microscopy of the nanowire-nanowire junctions created without water vapourization reveals no visible ablation or damage by laser-fusing (Fig. 3a).

Having in hand a method for manipulating, positioning and locally fusing nanowires, which we call laser nanowire assembly (LNA), we set out to build a three-dimensional nanowire heterostructure on the top surface of the chamber. We used two materials, $\mathrm{GaN}$ and $\mathrm{SnO}_{2} \cdot \mathrm{SnO}_{2}$ nanoribbons function as passive waveguides and $\mathrm{GaN}$ wires are subwavelength ultraviolet nanolasers, which may exploit one-dimensional quantum confinement of carriers to achieve high optical gain and a low lasing threshold ${ }^{25}$. Three $\mathrm{GaN}$ and two $\mathrm{SnO}_{2}$ wires were combined wire by wire, yielding an approximately $300 \mathrm{~nm}$ tall 'log cabin' consisting of waveguides and nanolasers and with six individually optically fused junctions (Fig. 3b). Each wire was trapped as it hovered on the lower surface of the chamber, raised to the lysine-coated top surface and then pushed onto the lysine coating. The wire, now docked to the top coverslip by one end, was then rotated about the tethered end by trapping and translation of its free end. Once the desired azimuthal angle with respect to other wires had been achieved, the wire was laid flat by bringing its free end to the surface (Fig. 1b).

Our positioning accuracy is limited significantly by brownian fluctuations. From the equipartition theorem, the r.m.s. transverse fluctuations of the GaN nanowires will be of the order of 10-60 nm (for laser powers ranging from 360 to $60 \mathrm{~mW}$, respectively). In addition, drift of the optical trap with respect to the stage and the use of visible optics with intrinsic diffraction limits further degrade positioning accuracy. For optimal accuracy, a closed-loop system exploiting fiduciary markers on the coverslip surface ${ }^{11}$ should be used. Also, provided that the wires are separated by more than half the wavelength of the illumination light, fitting of the point-spread function ${ }^{26,27}$ of light scattered from a wire should allow its continuous localization during assembly with improved resolution. Nevertheless, the positioning accuracy and the level of multilayer registration achieved using our laser trapping method are significantly better than those achievable with microfluidic channels or Langmuir-Blodgett techniques ${ }^{8,9}$. The use of holographic optical tweezers ${ }^{14}$, which are capable of simultaneously creating numerous independently steerable traps, should further facilitate manipulation and assembly of complex nanowire structures. For applications such as the construction of integrated optoelectronic switches, the water or other solvent present during LNA will need to be removed after assembly. However, for biomaterials applications in which semiconductor sensors, nanowaveguides and nanolasers are to be assembled next to living cells and tissues, the compatibility of LNA with aqueous
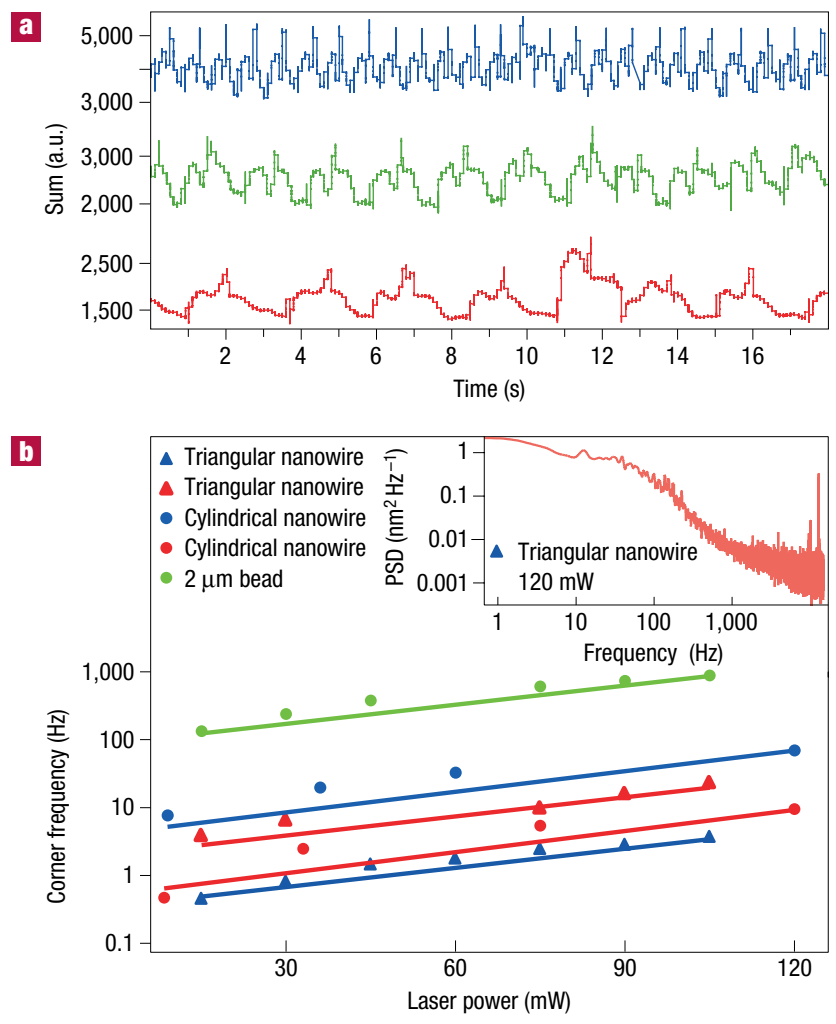

Figure 2 Oscillations of optically trapped semiconductor nanowires. a, Laser deflection versus time, illustrating the complex dynamics observed for rare, coupled $\mathrm{SnO}_{2}$ nanoribbons ( $\sim 1.5 \%$ of wires; see Supplementary Information). PSD sum signals shown are for trapping powers of $180 \mathrm{~mW}$ (red), $240 \mathrm{~mW}$ (green) and $360 \mathrm{~mW}$ (blue). b. Corner frequencies of PSD sum signals as a function of laser power, GaN cross-section and aspect ratio, which ranged from 60 to $300 \mathrm{~Hz}$ (GaN triangular (blue), 60; GaN triangular (red), 160; GaN cylindrical (blue), 300; GaN cylindrical (red), 100). Inset: power spectrum of PSD sum signal fluctuations for a GaN nanowire trapped at $120-\mathrm{mW}$ power. 


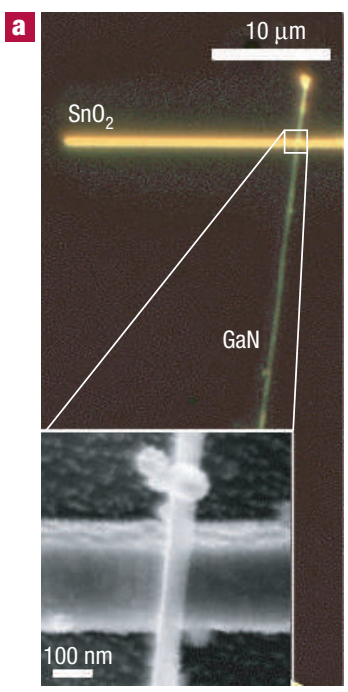

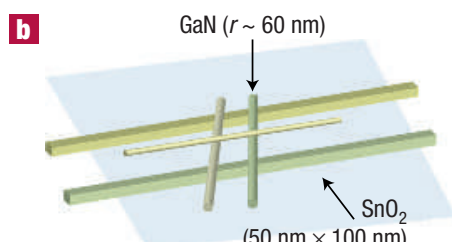

$(50 \mathrm{~nm} \times 100 \mathrm{~nm})$

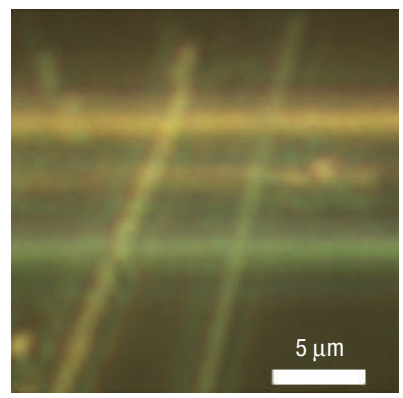

c

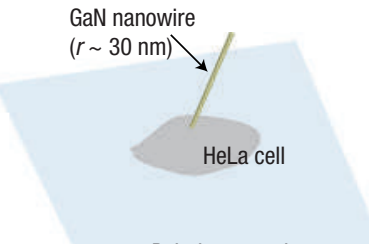

Poly-lys coated quartz

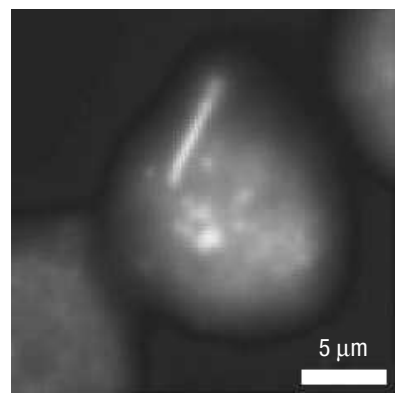

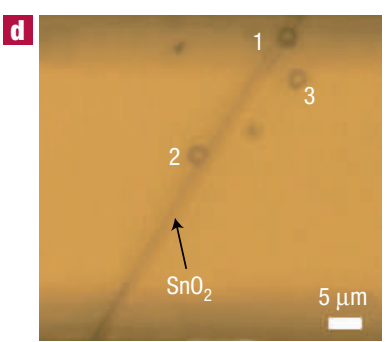

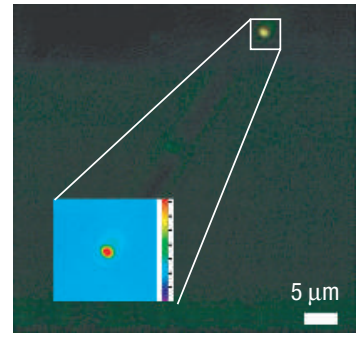

Figure 3 Demonstration of nanowire junctions and assemblies built using optical trapping. a, Darkfield image of a GaN nanowire laser-fused to a $\mathrm{SnO}_{2}$ nanoribbon. Inset: scanning electron micrograph of the fused junction, showing that it is not visibly ablated. Also visible are gold droplets generated from the gold-coated coverslip during laser-fusing. b. Schematic (top) and optical darkfield image (bottom) of a three-dimensional nanowire assembly consisting of $\mathrm{SnO}_{2}$ nanoribbons and GaN nanowires. c, Schematic (top) and optical darkfield image (bottom) of GaN nanowire brought to a human cervical cancer cell (HeLa) by optical trapping. Once positioned with respect to the cell, the wire was non-specifically attached to the cell's membrane by pushing the wire against the membrane for several seconds. $\mathbf{d}$, Brightfield (top) and fluorescence image (bottom) of 2- $\mu \mathrm{m}$ yellow-green fluorescent polystyrene beads (Molecular Probes) placed directly on or near a $\mathrm{SnO}_{2}$ nanoribbon resting on SU8 photoresist. Inset: wire-bead structure during ultraviolet excitation of the ribbon about $300 \mu \mathrm{m}$ from the bead. Fluorophores in bead 1, which touches the nanoribbon, are excited by the evanescent field created by waveguided photoluminescence generated elsewhere in the nanowire. Fluorescent beads that did not touch the ribbon, and instead were placed at radial distances of about $500 \mathrm{~nm}$ (bead 2) and about $5 \mu \mathrm{m}$ (bead 3), did not fluoresce. At these wavelengths, the 1/e decay distance of the evanescent field is less than $100 \mathrm{~nm}$.

environments is essential, as assembly will need to be done in water, at room temperature and at physiological ionic strength and $\mathrm{pH}$.

Optical imaging of biological samples benefits from a well-behaved and local excitation source. Using LNA, two complementary approaches can be immediately realized (Fig. 3c,d). A primary motivation for our choice of trapping wavelength $(1,064 \mathrm{~nm})$ was that living cells can tolerate limited infrared laser irradiation $^{28}$. As shown in Fig. 3c, optically trapped nanowires (here, a cylindrical GaN nanowire) can be positioned with respect to living cells. HeLa tissue culture cells were grown on lysine-coated quartz coverslips and chambers were assembled as before. It was possible to scan a trapped GaN nanowire across the cell membrane (see Supplementary Information, Video S3), and to place one end of the nanowire against the cell membrane and maintain the wire's position for arbitrary durations. GaN nanowires were used in these experiments as they have been demonstrated to waveguide and lase, suggesting their suitability as subwavelength illumination sources to image live cells and tissues by scanning the end of a GaN wire across a cell, or by pushing one end of the wire into the cell. In this way, local excitation of fluorophores, either by light radiating from the wire's end or through the evanescent field originating at the wire/water interface, could be achieved. As shown in Fig. 3d, local excitation of fluorophores by an evanescent field (here with a $1 / e$ decay distance of $\sim 100 \mathrm{~nm}$ ) from a $\mathrm{SnO}_{2}$ waveguide can be accomplished and suitably arranged $\mathrm{SnO}_{2}$ ribbons can thus be used as local sources of excitation light in various imaging and biosensing geometries. Evanescent excitation by the intrinsic white photoluminescence of the $\mathrm{SnO}_{2}$ ribbon and the waveguiding of optically coupled nanolaser emission has been demonstrated elsewhere ${ }^{29}$. Moreover, their small cross-section and very-high aspect ratio suggests their suitability to deliver extremely localized chemical, mechanical and electrical stimuli to cells, based on the construction of integrated assemblies next to a cell of interest. Therefore, in addition to the three-dimensional heterogeneous devices that can now be constructed from nanowires, LNA should facilitate new experiments for in situ characterization of biological materials.

\section{METHODS \\ INSTRUMENTATION}

The optical-tweezers setup (see Supplementary Information, Fig. S1) is built around a Nikon $\times 60$ microscope objective (CFI PLAN APO 60X WI NA 1.20 WD 0.22MM, Nikon part number 93109). A 1.6-mm-diameter infrared beam (1,064 nm, 2W Laser, Coherent COMPASS 1.064 2000N) was expanded to $16 \mathrm{~mm}$ using a beam expander (BE10; Thorlabs), overfilling the back focal plane of the objective. A specimen in the object plane can be moved coarsely along $x$ and $y$ with a homemade manual translation stage (machined out of invar, with a height of $6 \mathrm{~mm}$ ), and finely with a $x y z$-piezo stage (Nano-UHV100; Mad City Lab, Madison, Wisconsin, USA). The piezo stage has $100 \mu \mathrm{m}$ of travel along $x$ and $y$ directions and $25 \mu \mathrm{m}$ in the $z$ direction. The stage was interfaced to the computer through a DT9834 USB module (Data Translation) and controlled through software written in Visual $\mathrm{C}++$. Poly-L-lysine was purchased from Ted Pella, and the quartz coverslips were obtained from SPI (thickness number 1 (0.15-0.18 mm thick), $25 \mathrm{~mm}$ diameter, part number 01019T-AB). Scanning electron microscopy experiments were performed with an FEI Strata DB235, set to $5 \mathrm{keV}$ and a magnification of 35,000.

\section{EXPERIMENTAL CHAMBERS AND NANOWIRE SYNTHESIS}

Experimental chambers were prepared as follows. Nanowires, chemically grown as described elsewhere $\left(\mathrm{GaN}, \mathrm{SnO}_{2}, \mathrm{ZnO}, \mathrm{Si}\right.$ and $\mathrm{Ag}$, reviewed in ref. 4), were suspended in deionized water by means of sonication. Several microlitres of the nanowire-water suspension were transferred by means of a pipette into a flow chamber consisting of a glass slide and quartz coverslip covered with $1.7 \mathrm{~nm}$ of gold (deposited by standard thermal evaporation techniques) or a thin lysine film (for compatibility with living cells). The edges of the glass slide and the 
quartz coverslip were then sealed with a $100-\mu \mathrm{m}$-thick adhesive tape. Quartz was used as the coverslip material to minimize autofluorescence during subsequent optical excitation of assembled structures.

Received 10 September 2005; accepted 22 November 2005; published 22 January 2006.

\section{References}

1. Wang, W. U., Chen, C., Lin, K. H., Fang, Y. \& Lieber, C. M. Label-free detection of small-molecule-protein interactions by using nanowire nanosensors. Proc. Natl Acad. Sci. USA 102 3208-3212 (2005)

2. Karnik, R. et al. Electrostatic control of ions and molecules in nanofluidic transistors. Nano Lett. 5, 943-948 (2005).

3. Sirbuly, D. J., Law, M., Yan, H. Q. \& Yang, P. D. Semiconductor nanowires for subwavelength photonics integration. J. Phys. Chem. B 109, 15190-15213 (2005).

4. Xia, Y. N. et al. One-dimensional nanostructures: Synthesis, characterization, and applications. $A d v$ Mater. 15, 353-389 (2003).

5. Kuykendall, T. et al. Crystallographic alignment of high-density gallium nitride nanowire arrays. Nature Mater. 3, 524-528 (2004).

6. Smith, P. A. et al. Electric-field assisted assembly and alignment of metallic nanowires. Appl. Phys. Lett. 77, 1399-1401 (2000).

7. Chen, M. et al. Tuning the response of magnetic suspensions. Appl. Phys. Lett. 82, 3310-3312 (2003).

8. Messer, B., Song, J. H. \& Yang, P. D. Microchannel networks for nanowire patterning. J. Am. Chem. Soc. 122, 10232-10233 (2000).

9. Yang, P. Nanotechnology: Wires on water. Nature 425, 243-244 (2003).

10. Law, M., Goldberger, J. \& Yang, P. D. Semiconductor nanowires and nanotubes. Annu. Rev. Mater. Res. 34, 83-122 (2004)

11. Nugent-Glandorf, L. \& Perkins, T. T. Measuring 0.1-nm motion in $1 \mathrm{~ms}$ in an optical microscope with differential back-focal-plane detection. Opt. Lett. 29, 2611-2613 (2004).

12. Korda, P., Spalding, G. C., Dufresne, E. R. \& Grier, D. G. Nanofabrication with holographic optical tweezers. Rev. Sci. Instrum. 73, 1956-1957 (2002).

13. Ashkin, A., Dziedzic, J. M., Bjorkholm, J. E. \& Chu, S. Observation of a single-beam gradient force optical trap for dielectric particles. Opt. Lett. 11, 288-290 (1986).

14. Grier, D. G. A revolution in optical manipulation. Nature 424, 810-816 (2003).

15. Sato, S., Harada, Y. \& Waseda, Y. Optical trapping of microscopic metal particles. Opt. Lett. 19, 1807-1809 (1994)

16. Svoboda, K. \& Block, S. M. Optical trapping of metallic Rayleigh particles. Opt. Lett. 19, 930-932 (1994).
17. Friese, M. E. J., Nieminen, T. A., Heckenberg, N. R. \& Rubinsztein-Dunlop, H. Optical alignment and spinning of laser-trapped microscopic particles. Nature 394, 348-350 (1998).

18. Yu, T., Cheong, F. C. \& Sow, C. H. The manipulation and assembly of $\mathrm{CuO}$ nanorods with line optical tweezers. Nanotechnol. 15, 1732-1736 (2004)

19. Gauthier, R. C. Theoretical investigation of the optical trapping force and torque on cylindrical micro-objects. J. Opt. Soc. Am. B 14, 3323-3333 (1997).

20. Gauthier, R. C., Ashman, M. \& Grover, C. P. Experimental confirmation of the optical-trapping properties of cylindrical objects. Appl. Opt. 38, 4861-4869 (1999).

21. Shelton, W. A., Bonin, K. D. \& Walker, T. G. Nonlinear motion of optically torqued nanorods. Phys. Rev. E 71, 036204 (2005).

22. Kress, H., Stelzer, E. H. K. \& Rohrbach, A. Tilt angle dependent three-dimensional-position detection of a trapped cylindrical particle in a focused laser beam. Appl. Phys. Lett. 84, 4271-4273 (2004).

23. Rubinsztein-Dunlop, H., Nieminen, T. A., Friese, M. E. J. \& Heckenberg, N. R. Optical trapping of absorbing particles. Adv. Quant. Chem. 30, 469-492 (1998).

24. Wu, Y. Y. \& Yang, P. D. Melting and welding semiconductor nanowires in nanotubes. Adv. Mater. 13, 520-523 (2001).

25. Johnson, J. C., Yan, H. Q., Yang, P. D. \& Saykally, R. J. Optical cavity effects in ZnO nanowire lasers and waveguides. J. Phys. Chem. B 107, 8816-8828 (2003).

26. Thompson, R. E., Larson, D. R. \& Webb, W. W. Precise nanometer localization analysis for individual fluorescent probes. Biophys. J. 82, 2775-2783 (2002).

27. Gelles, J., Schnapp, B. J. \& Sheetz, M. P. Tracking kinesin-driven movements with nanometre-scale precision. Nature 331, 450-453 (1988).

28. Ashkin, A., Dziedzic, J. M. \& Yamane, T. Optical trapping and manipulation of single cells using infrared-laser beams. Nature 330, 769-771 (1987).

29. Sirbuly, D. J. et al. Optical routing and sensing with nanowire assemblies. Proc. Natl Acad. Sci. USA 102, 7800-7805 (2005).

\section{Acknowledgements}

We thank J. Choy, J. Feigelman, C. Hodges, the Yang Lab (A. Tao, H. Yan, A. Hochbaum and D. Sirbuly) and M. van Duijn. P.J.P. thanks the NSF for Graduate Research Support and H.S. thanks the Hertz Foundation. This work was supported in part by the University of California, Berkeley (J.L.), the Beckman Foundation and the Department of Energy (P.Y.).

Correspondence and requests for materials should be addressed to J.L. or P.Y.

Supplementary Information accompanies this paper on www.nature.com/naturematerials.

Competing financial interests

The authors declare that they have no competing financial interests.

Reprints and permission information is available online at http://npg.nature.com/reprintsandpermissions/ 